\title{
Análise da relação entre NDVI e a temperatura da superfície terrestre como técnica no planejamento urbano dos municípios
}

\author{
Análisis de la relación entre NDVI y temperatura de la \\ superficie de la tierra como técnica en la planificación urbana de \\ los municipios
}

\section{Analysis of the relationship between NDVI and land surface temperature as a technique in the urban planning of the} counties

\author{
Dhonatan Diego Pessi \\ dhonatan.pessi@gmail.com
}

Universidade Federal de Rondonópolis, UFR, Rondonópolis, MT

Cauê Felipe Pimentel

cauefp@hotmail.com

Universidade Federal de Rondonópolis, UFR, Rondonópolis, MT

Anny Keli Aparecida Alves Cândido

hannykeli@gmail.com

Universidade Federal de Rondonópolis, UFR, Rondonópolis, MT

Pedro Lopes Miranda Junior

fuzlopes@gmail.com

Universidade Federal de Rondonópolis, UFR, Rondonópolis, MT

Normandes Matos da Silva normandes32@gmail.com

Universidade Federal de Rondonópolis, UFR, Rondonópolis, MT

Resumo: O rápido crescimento populacional e a grande exploração dos recursos naturais implicam em grandes modificações no ambiente ocasionando impactos ambientais, dentre eles o aumento da temperatura média da terra. Nesse sentido, diversos estudos estão sendo desenvolvidos buscando entender a influência desses impactos sobre a saúde dos organismos. O objetivo deste trabalho foi analisar a relação entre os valores de NDVI com as variações da temperatura da superfície, a fim de entender a influência da vegetação na variação termal urbana de cidades de médio porte. Esse trabalho foi desenvolvido com imagens de satélites disponibilizadas de forma gratuita pelo Serviço Geológico Americano e o processamento foi realizado em linguagem R. Para gerar o NDVI optou-se por usar imagem Sentinel-2 com resolução espacial de 10 metros e para o cálculo da temperatura utilizou-se uma cena Landsat8 
com resolução espacial de 30 metros pelo fato da mesma possuir a banda termal. Existe uma relação moderada negativa nas variações da temperatura conforme a ausência ou presença de vegetação na superfície terrestre $(\mathrm{p}<0.05)$. O NDVI e o LST exibiram padrões de distribuição espacial inversamente proporcional. A compreensão dos efeitos da mudança da cobertura da terra permite maior agilidade na tomada de decisão por parte dos gestores públicos, a fim de mitigar os efeitos do calor urbano e a formação de ilhas de calor com a inserção de vegetação em áreas densamente ocupadas.

Palavras-chave: Programação R; ilhas de calor urbana; arborização urbana; gestão municipal.

Resumen: El rápido crecimiento poblacional y la gran explotación de los recursos naturales implican en grandes modificaciones en el ambiente ocasionando impactos ambientales, entre ellos el aumento de la temperatura media de la tierra. En ese sentido, diversos estudios están siendo desarrollados buscando entender la influencia de esos impactos sobre la salud de los organismos. El objetivo de este trabajo fue analizar la relación entre los valores de NDVI con las variaciones de la temperatura de la superficie, a fin de entender la relación de la vegetación en la variación termal urbana de ciudades de mediano porte. Este trabajo fue desarrollado con imágenes de satélites disponibles de forma gratuita por el Servicio Geológico Americano y el procesamiento fue realizado en lenguaje R. Para generar el NDVI se optó por usar imagen Sentinel-2 con resolución espacial de 10 metros y para el cálculo de la temperatura se utilizó una escena Landsat 8 con resolución espacial de 30 metros por el hecho de esta poseer la banda termal. Existe una relación moderada negativa en las variaciones de la temperatura según la ausencia o presencia de vegetación en la superficie terrestre $(p<0.05)$. El NDVI y el LST mostraron patrones de distribución espacial inversamente proporcional. La comprensión de los efectos del cambio de la cobertura de la tierra permite mayor agilidad en la toma de decisión por parte de los gestores públicos, a fin de mitigar los efectos del calor urbano y la formación de islas de calor con la inserción de vegetación en áreas densamente ocupadas.

Palabras-clave: Programación R; islas de calor urbano; arborización urbana; gestión municipal.

\begin{abstract}
The fast population growth and the great exploitation of the natural resources, imply in great modifications in the environment causing environmental impacts, among them, the average temperature increases of the Earth. In that sense, several studies tried to understand the influence of these impacts on the health of the organisms. This study aimed to analyze the relationship between NDVI values and surface temperature variations, in order to understand the relation of vegetation in the urban thermal variation of midsize cities. This work used satellites free available images by the American Geological Survey and the processing employed R language. In order to generate the NDVI, we chose to use Sentinel-2 image with $10 \mathrm{~m}$ spatial resolution, and for the calculation of the temperature, we used a Landsat 8 scene with $30 \mathrm{~m}$ spatial resolution, once it has thermal band. There is a moderate negative relation in the temperature variations according to the absence or presence of vegetation in the terrestrial surface $(\mathrm{p}<0.05)$. The NDVI and LST exhibited inversely proportional spatial distribution. Understanding the effects of changing land cover allows greater agility in decision making by public managers in order to mitigate the effects of urban heat and the formation of heat islands by vegetation insertion in densely occupied areas.
\end{abstract}

Keywords: R programming; urban heat islands; urban afforestation; municipal management. 


\section{INTRODUÇÃO}

A relação entre o padrão de cobertura da terra com o aumento da temperatura em áreas urbanas implica em impactos sobre o ambiente, bem como sobre a saúde humana (GORGANI; PANAHI; REZAIE, 2013). Na medida em que a população urbana continua em rápido crescimento, aumenta a probabilidade de ocorrer maior remoção da cobertura natural da terra, alterando os recursos hídricos locais, substituindo-os por materiais urbanos comuns, como asfalto, concreto e metal, o que promove a redução da evapotranspiração, torna o escoamento superficial mais intenso e rápido, incluindo o aumento e armazenamento de calor sensível e a redução da qualidade do ar e da água (YUE et al., 2007).

Em termos de mensuração do ambiente térmico urbano, o sensoriamento remoto fornece medições da temperatura da superfície radiante, incluindo medições de energia refletora nas porções vermelha e infravermelha próxima do espectro eletromagnético, que podem ser utilizadas para avaliar a cobertura vegetal urbana, em termos de conforto térmico (YUE et al., 2007).

A temperatura da superfície da terra (LST, Land Surface Temperature) é um dos fatoreschave na física do processo de superfície do planeta, combinando interações superfícieatmosfera e os fluxos de energia entre a atmosfera e o solo (GORGANI; PANAHI; REZAIE, 2013). É um fator importante que reflete mudanças ambientais da superfície e influi nos processos físicos e químicos em áreas urbanas (DENG et al., 2018).

Existem muitos estudos que utilizaram o Índice de Vegetação por Diferença Normalizada (NDVI, Normalized Difference Vegetation Index), para determinar a relação entre a temperatura das superfícies urbanas e temperatura térmica a partir de imagens de satélite (BEZERRA; MORAES; SOARES, 2018; BEZERRA et al., 2018; GODOY; BAPTISTA; ALMEIDA, 2009; COLA; LOPES; BAZAN, 2017). Valores mais altos de NDVI indicam alta fração de vegetação (MWANGI; KARANJA; KAMAU, 2018), e a correlação entre LST e NDVI é negativa devido ao processo de evapotranspiração, que reduz a temperatura da superfície (VOOGT; OKE, 2003). É possível também, com as porções vermelha e infravermelha próxima do espectro eletromagnético, quantificar a extensão e as mudanças nas condições da vegetação urbana. A visão sinótica do mosaico urbano fornecida por satélites e sensores aéreos é um complemento importante para as medições in situ de variáveis físicas, ambientais e socioeconômicas em ambientes urbanos (YUE et al., 2007; SMALL, 2004).

O objetivo deste trabalho foi apresentar resultados de uma análise da relação entre os valores de NDVI com as variações da temperatura da superfície terrestre na mancha urbana de Rondonópolis (MT), região central do Brasil de clima subtropical úmido (cwa), a fim de entender a influência da vegetação na variação termal urbana de cidades de médio porte. 


\section{MATERIAL E MÉTODOS}

\section{Área de estudo}

A mancha urbana da cidade de Rondonópolis está localizada no sudeste do estado

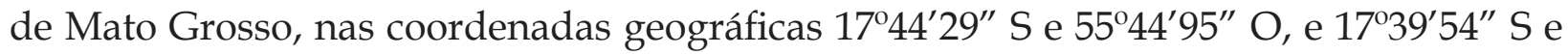
5350'64" O, e dista 212 km da capital, Cuiabá (Fig. 1).

Figura 1: Localização do município de Rondonópolis, MT, e delimitação da mancha urbana com área construída.

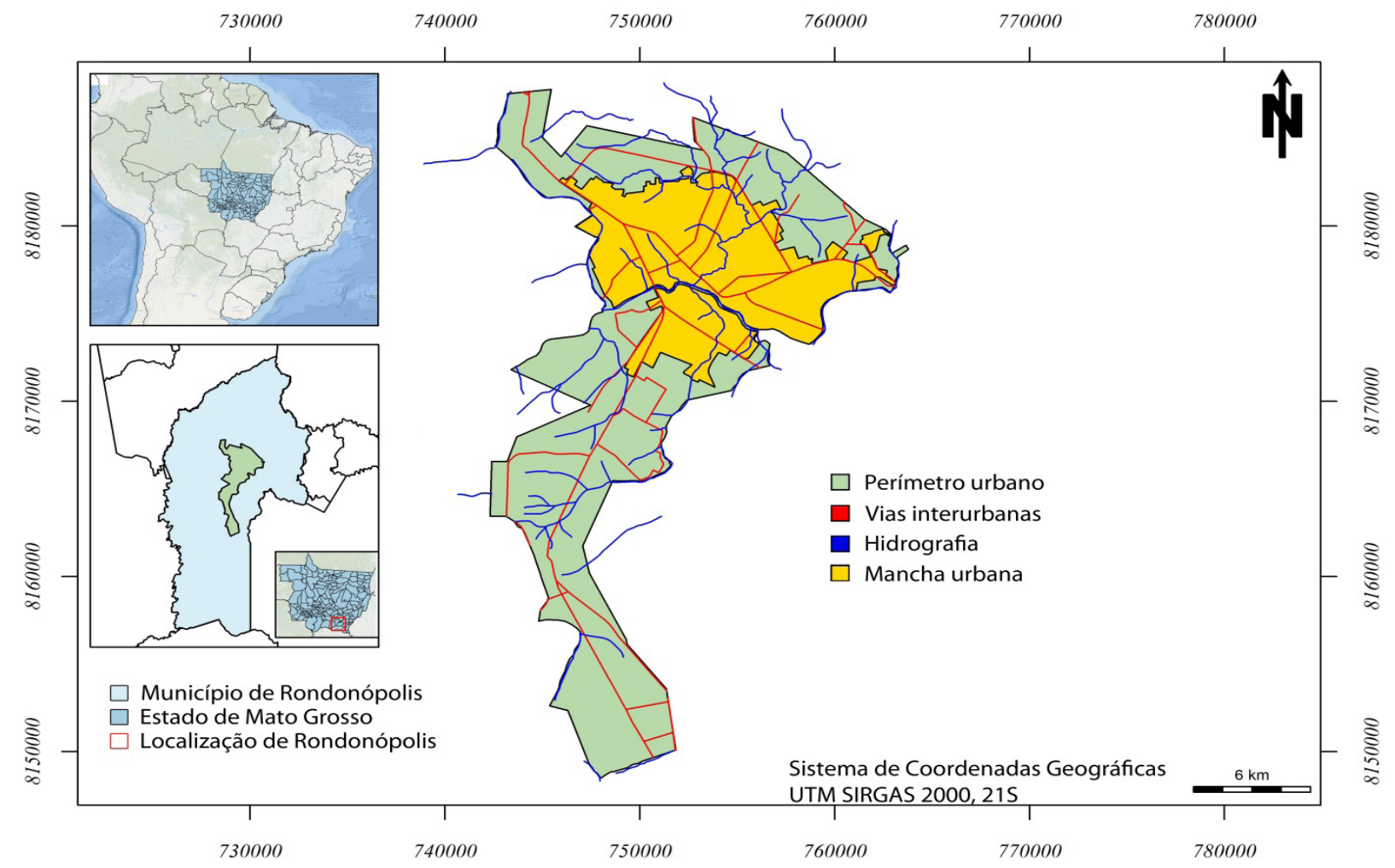

Fonte: IBGE disponível em: https:/ / downloads.ibge.gov.br/downloads_geociencias.htm. Confecção: D.D. Pessi, 2018.

O município possui um padrão climático do tipo Cwa (subtropical úmido) (PEEL; FINLAYSON; MCMAHON, 2007), com temperatura média anual de $21^{\circ} \mathrm{C}$, com média máxima de $32,6^{\circ} \mathrm{C}$ e média mínima de $18,6^{\circ} \mathrm{C}$ (SOUZA et al., 2013). A vegetação é formada por diversas tipologias de Cerrado e Floresta Estacional. As altitudes estão na faixa de 200 a 300 m.s.n.m. As classes pedológicas dessa região são principalmente Latossolos, Neossolos, Argissolos e Cambissolos (PINHEIRO; CASTRO; MARTINS, 2008). 


\section{Aquisição das imagens de satélite}

Foram utilizadas cenas de dois satélites ${ }^{1}$ : Sentinel-2, instrumento imageador MSI e Landsat-8, instrumento imageador TIRS. Para o cálculo de NDVI, utilizou-se de uma cena do satélite Sentinel-2 com resolução espacial de 10 metros datado de 14/maio/2018 (USGS, 2018a). Para este caso, foi usado o satélite Sentinel-2 por se tratar do satélite de melhor resolução espacial gratuito. Já no cálculo de temperatura, foi usado uma cena do satélite Landsat-8 (USGS, 2018b), com resolução espacial de 30 metros, datado de 16/maio/2018. O cálculo de temperatura ocorreu mediante o uso das bandas 10 do satélite Landsat-8, pois o Sentinel-2 não possui banda termal.

\section{Índice de Vegetação por Diferença Normalizada (NDVI)}

O cálculo do índice de NDVI envolve a razão normalizada entre a diferença e a soma das bandas do infravermelho próximo (NIR) e o vermelho (RED) (ROUSE et al., 1973). Esse índice normaliza a razão simples para o intervalo de -1 a +1 , sendo que as áreas com vegetação mais intensa se aproximam de +1 , e os valores próximos de 0 geralmente representam solo exposto. Os valores negativos indicam superfícies com água. Estas duas bandas espectrais foram escolhidas porque são as mais afetadas pela absorção de clorofila na vegetação de folhas verdes e pela densidade da vegetação na superfície. O NDVI é calculado pela equação (1):

$$
N D V I=\frac{N I R-R E D}{N I R+R E D}
$$

Onde:

NIR = Infravermelho próximo $(780-2.500 \mathrm{~nm})$

$R E D=$ Vermelho (625-740 $\mathrm{nm})$

O cálculo do índice de NDVI foi realizado a partir das bandas NIR e RED extraídas da cena do satélite Sentinel-2. Após realizar a separação das bandas de interesse para o cálculo de vegetação, estas utilizaram um script do software livre R 3.5.0 (R CORE TEAM, 2018), para o cálculo do índice e elaboração dos gráficos. As áreas escolhidas para análise da cobertura vegetal do solo urbano foram obtidas a partir de quatro unidades amostrais (Fig. 2), nas quais foi realizado o levantamento da quantidade de área construída e de vegetação (Tab. 1). As unidades amostrais escolhidas compreendem porções da região central da cidade (unidades amostrais 1, 2 e 3) e regiões periféricas da mancha urbana (unidade amostral 4). 
Figura 2 - Localização das unidades amostrais utilizadas no estudo.
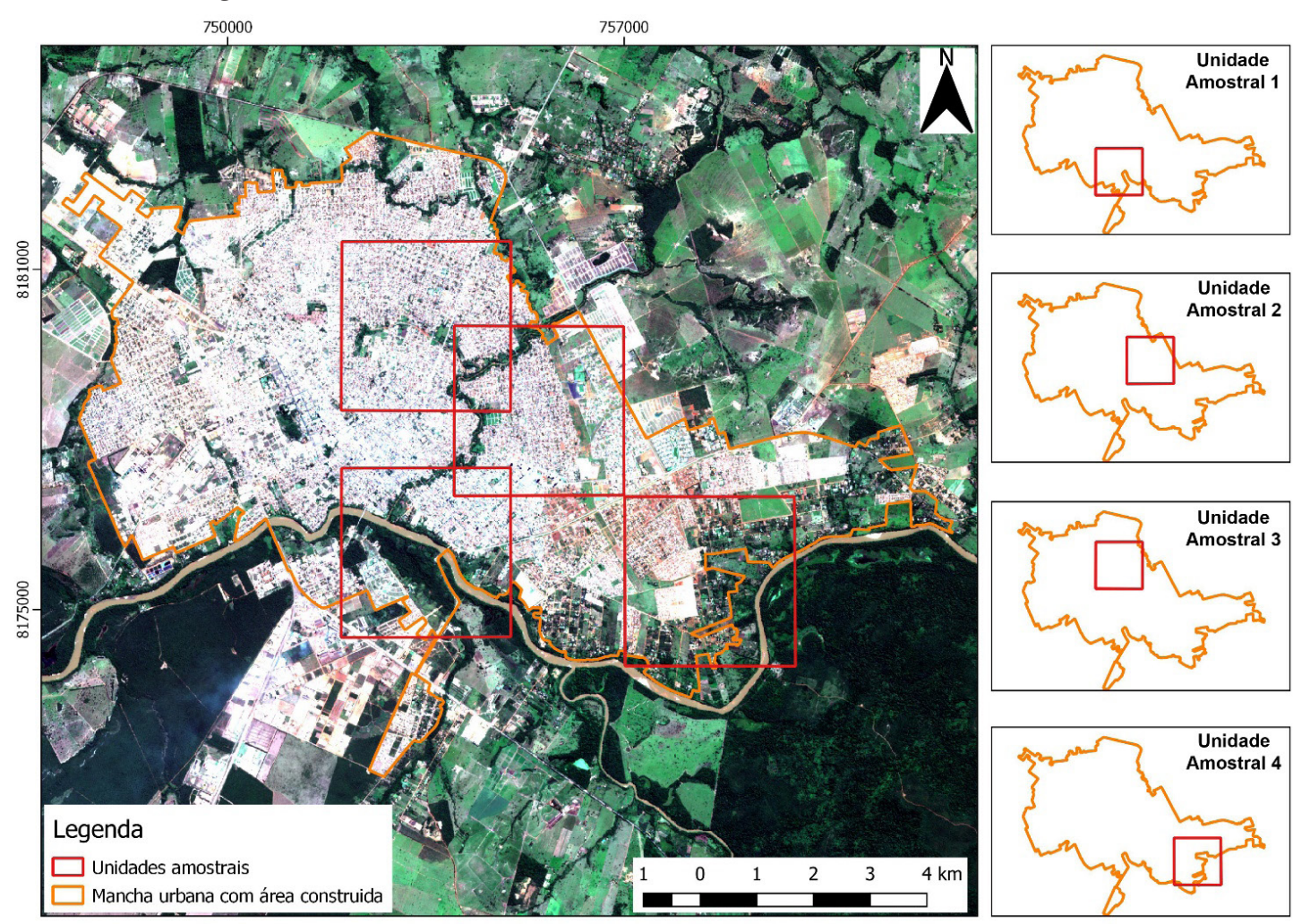

Fonte: imagem Sentinel2 de 14/05/2018, 16 bits e resolução espacial de 10 m.

Tabela 1: Levantamento em hectares da área construída e cobertura vegetal nas unidades amostrais.

\begin{tabular}{c|c|c|c|c}
\hline Unidade Amostral & 1 & 2 & 3 & 4 \\
\hline Cobertura Vegetal (ha) & 255 & 157 & 87 & 334 \\
\hline Área Construída (ha) & 464 & 709 & 814 & 330 \\
\hline
\end{tabular}

Fonte: os autores.

\section{Cálculo de temperatura da superfície terrestre (LST)}

O cálculo de temperatura se deu a partir da banda termal (TIRS - Thermal Infrared Sensor) do satélite Landsat-8. Para realizar o cálculo do LST, primeiramente deve-se converter os valores dos pixels da imagem ou os Números Digitais (ND) do sensor TIRS, em valores de refletância obtidos no topo da atmosfera (TOA, Top of Atmosphere) usando a equação (2):

$$
L \lambda=M_{L} Q_{c a l}+A_{L}
$$

Onde:

$L_{\lambda}$ é o valor da radiância espectral no topo da atmosfera (Watts $/\left(\mathrm{m} 2{ }^{*} \operatorname{srad}^{*} \mu \mathrm{m}\right)$ ) $M_{L}$ é o fator multiplicativo escalonado específico constante no arquivo de metadados (RADIANCE_MULT_BAND_x, onde $x$ é o número da banda)

$Q_{c a l}$ é o produto padrão quantificado e calibrado por valores de pixel (ND) 
$A_{L}$ é o fator aditivo escalonado específico constante no arquivo de metadados (RADIANCE_ADD_BAND_x, onde $x$ é o número da banda).

Na próxima etapa, converte-se os valores de refletância em temperatura de brilho do satélite, que neste caso é em Kelvin. Isso é feito através da equação (3) e também necessita de alguns dados do metarquivo:

$$
T=K_{2} / l_{n}\left(\frac{K_{1}}{L_{\lambda}}+1\right)
$$

Onde:

T é a temperatura de brilho em Kelvin (K)

$L_{\lambda}$ é a refletância no topo da atmosfera (Watts $/\left(\mathrm{m}^{2} *\right.$ srad * $\left.\left.\mu \mathrm{m}\right)\right)$

$K_{1}$ é a constante de conversão $K 1$ específica para cada banda, também denominada de constante térmica, disponível no arquivo de metadados (K1_CONSTANT_BAND_x, onde $x$ é o número da banda)

$K_{2}$ é a constante de conversão $K 2$ específica para cada banda, também denominada de constante térmica, disponível no arquivo de metadados (K2_CONSTANT_BAND_x, onde x é o número da banda).

Após a conversão de valores de refletância em dados de temperatura, converte-se os dados de temperatura Kelvin em graus Celsius de acordo com a equação (4):

$$
T_{C}=T_{K}-273
$$

Onde:

$T_{c}$ é a temperatura de brilho em graus Celsius $\left({ }^{\circ} \mathrm{C}\right)$

$T_{k}$ é a temperatura de brilho em Kelvin (K).

\section{Análise dos dados}

A partir dos resultados dos cálculos de NDVI e de LST foi possível gerar dados matriciais, com informações de valores de NDVI e LST para cada pixel do raster. A partir daí, criou-se um data frame com duas colunas, uma com as informação de NDVI e outra com as informações de LST, para cada uma das unidades amostrais. As informações de NDVI e LST que foram extraídas de cada unidade amostral são referentes aos mesmos pontos de pixel, ou seja, o R extraiu para o mesmo ponto do raster de NDVI a informação correspondente do LST para o mesmo ponto. O data frame foi criado para que em cada linha o valor informado de NDVI corresponda ao de LST. Uma vez com estas informações tabuladas em matrizes no R, foi possível realizar inferências estatísticas - correlação de Pearson e regressão linear simples. A importação e a manipulação dos dados para a elaboração das análises estatísticas se deram novamente no R. 


\section{RESULTADOS E DISCUSSÃO}

\section{Análise visual dos padrões de contraste da Temperatura da Superfície Terrestre (LST) e do NDVI}

É observado na Figura 3 que o LST e o NDVI exibiram padrões de distribuição espacial opostos. Nas imagens a1, b1, c1 e d1, observa-se que as áreas com valores superiores a 0.5 de NDVI referem-se à vegetação mais densa e com maior IAF (índice de área foliar) desenvolvida. Em contraste com estas áreas, nas imagens a2, b2, c2 e d2, constata-se que os valores de LST caem até $5^{\circ} \mathrm{C}$ em relação aos espaços entre a vegetação e áreas edificadas, asfalto e outros elementos, ou seja, a presença de vegetação de maior porte exerce um efeito no microclima. No nível pontual, o LST nas áreas centrais formou o fenômeno de ilha de calor, que pode estar relacionado à ausência de vegetação.

Os dados de temperatura de LST mostraram que as temperaturas mais baixas foram de $24^{\circ} \mathrm{C}$ a $25^{\circ} \mathrm{C}$ nas regiões periféricas da cidade de Rondonópolis. As temperaturas mais altas foram de $32^{\circ} \mathrm{C}$ a $31^{\circ} \mathrm{C}$ e estavam representadas nas áreas centrais da mancha urbana, onde há mais área construída. A temperatura média geral foi de $28,5^{\circ} \mathrm{C}$. Os valores encontrados para o NDVI foram desde valores negativos a valores positivos. Na imagem a1, os valores negativos de -0.2 representam o rio Vermelho que cruza a região central da cidade de Rondonópolis. Ademais, os valores estão representados a partir de 0.0, que caracteriza a ausência total de vegetação. Os maiores valores de NDVI encontrados foram de 0.8 , com sua maior representação na imagem a1.

Em trechos da periferia da área de estudo, próximos ao rio Vermelho e em áreas com propriedades usadas como chácaras (unidade amostral 1 e 4), o LST formou uma área de baixos valores de temperatura, com valores médios de $27,8^{\circ} \mathrm{C}$ e $27,5^{\circ} \mathrm{C}$ respectivamente às unidades amostrais 1 e 4 . As temperaturas mais altas estão associadas aos prédios urbanos e principais vias de transporte, compostos de materiais não porosos, como metal, asfalto e concreto (LO; QUATTROCHI; LUVALL, 1997). As unidades amostrais 2 e 3 obtiveram médias de temperatura de $29,4^{\circ} \mathrm{C}$ e $29,5^{\circ} \mathrm{C}$, respectivamente. Na periferia da mancha urbana, há um contraste urbano-rural, pois neste espaço existem pequenas chácaras urbanas, o que indica a saída das ilhas de calor da faixa mais central para as áreas periféricas.

Observa-se nas imagens b2 e c2, as quais obtiveram os maiores valores de área construída, com 709 hectares e 814 hectares respectivamente (Tab. 1), que o LST do centro urbano é maior do que na periferia. Além disso, o LST formou uma área de alto valor nas regiões com maior quantidade de área construída, e em outras áreas de construção intensiva, mostrando um fenômeno regular de ilha de calor urbana (DENG et al., 2018).

Em outros trabalhos, como o de Deng et al. (2018), foi verificado que no verão o efeito de ilha de calor urbana, por conta de áreas com edificações e áreas industriais, é mais notável. No entanto, além das ilhas de calor causadas pelo ambiente urbano convencional, muitas zonas de alta temperatura com diferentes formas e tamanhos foram observadas em regiões periféricas. O mesmo foi observado para as regiões periféricas de Rondonópolis, onde, como na unidade amostral 4, com presença de chácaras com pouca ou nenhuma vegetação, 
Figura 3: padrão espacial e relação entre ambiente térmico urbano e da cobertura vegetal na área urbana da cidade de Rondonópolis, MT. As imagens a1, b1, c1 e d1 representam o índice NDVI das unidades amostrais 1, 2, 3 e 4. As imagens a2, b2, c2 e d2 representam a temperatura LST das unidades amostrais 1 , 2,3 e 4 . As demarcações em linhas na cor preta representam os fragmentos de vegetação com valores de NDVI maiores do que 0.5 e áreas onde a temperatura é inferior a $28^{\circ} \mathrm{C}$.
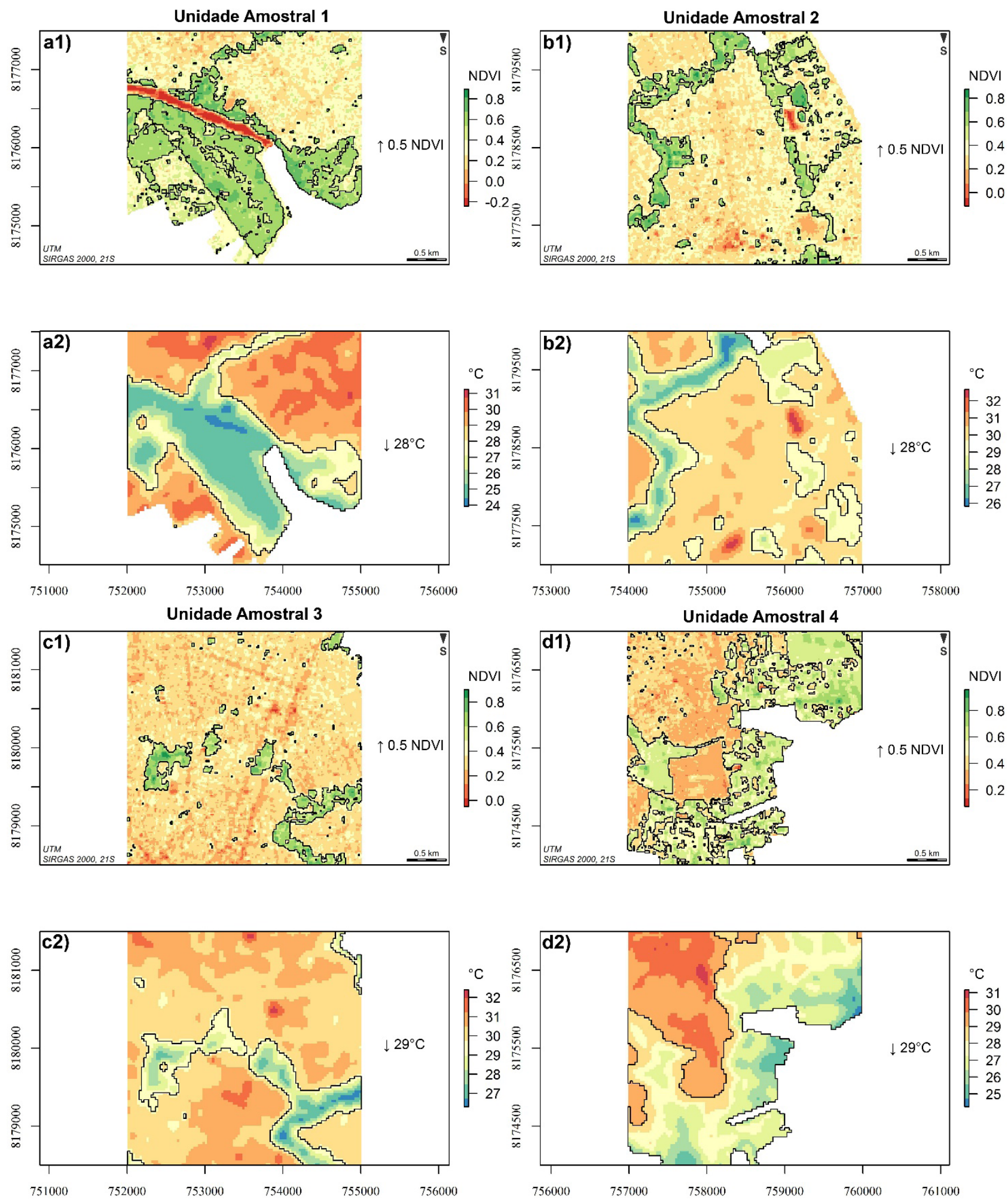

Fonte: os autores. 
existem fragmentos de tamanhos variados com altas temperaturas. Os mesmos autores relatam que resultados de pesquisas existentes sobre a relação entre LST e NDVI podem variar consideravelmente sob combinações de diferentes tipos de superfície subjacentes (DENG et al., 2018).

Também foi observado por Yue et al. (2007) uma forte relação de áreas arborizadas com baixas taxas de LST, assim como foi observado para o caso de Rondonópolis. O maior valor médio de LST é devido à grande quantidade de calor por conta do centro urbano sem vegetação, caso este semelhante às regiões centrais e com maior quantidade de área construída (Fig. 3 c2). Os tipos de uso e cobertura de uso comercial ou serviços também mostram maior LST que, juntamente com o tipo industrial, formam um dos efeitos do UHI (YUE et al., 2007). Ao mesmo tempo, o parque ou terrenos verdes exibem o maior valor médio de NDVI devido à predominância de árvores, grama e outros tipos de uso e cobertura de vegetação. A cobertura vegetal em parques ou outro tipo de terreno verde, sugere taxas comparativamente mais altas de evapotranspiração e favorecimento da troca de calor latente entre a superfície e a atmosfera, quando comparada com lugares com menor quantidade de vegetação (WILSON et al., 2003)

Uma análise de regressão linear simples foi realizada para todas as unidades amostrais, sendo a LST variável dependente, e o NDVI a variável independente. A Figura 4 mostra os resultados do gráfico de dispersão e a linha de regressão e os valores de R2 e correlação de Pearson (r) constam da Tabela 2.

Figura 4: Gráfico de dispersão da análise de regressão linear simples entre os valores de LST e NDVI para cada unidade amostral analisada.
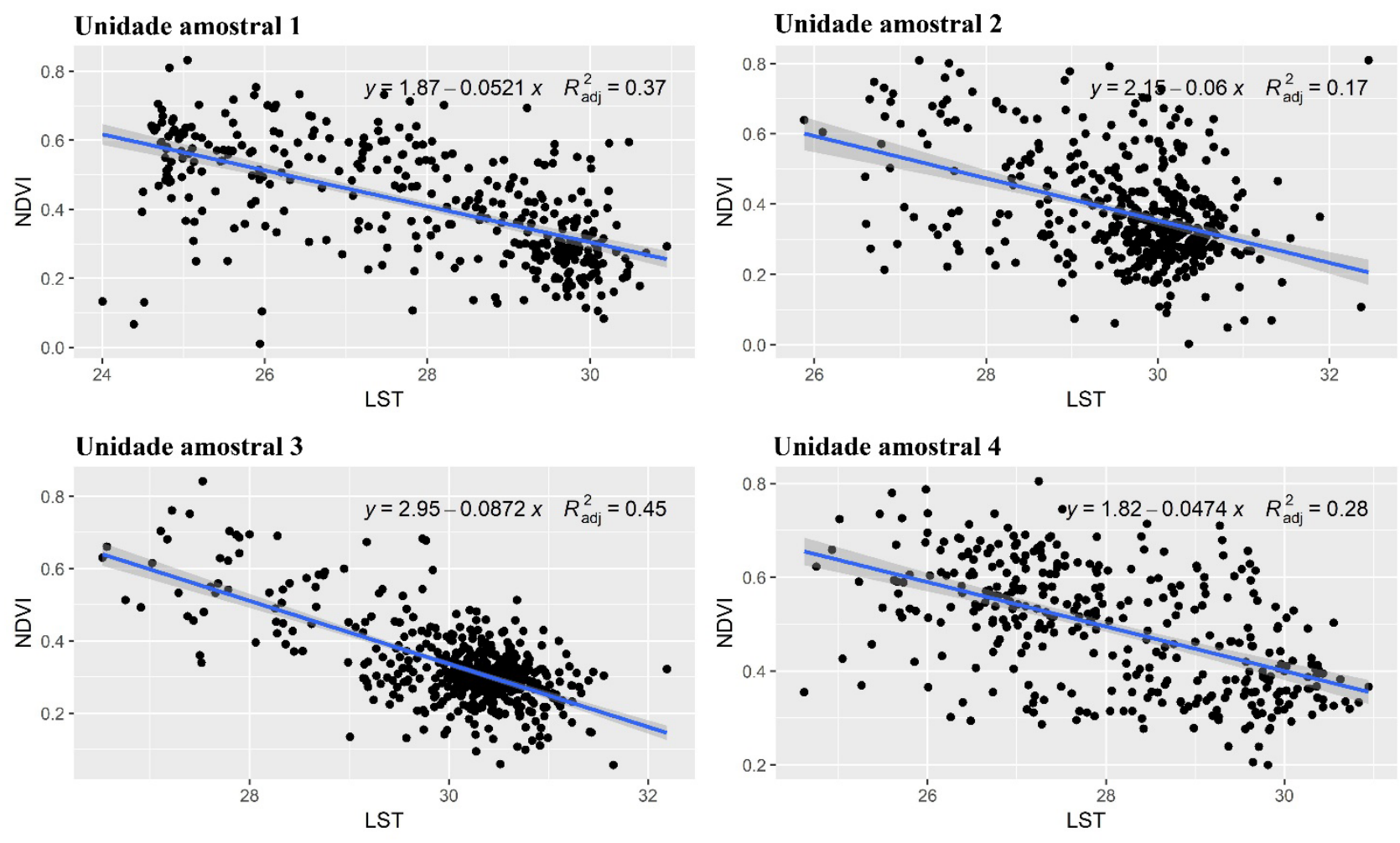

Fonte: Os autores. 
Tabela 2: Valores de R2 e correlação de Pearson (r) de LST com NDVI.

\begin{tabular}{l|c|c|c|c}
\hline $\begin{array}{l}\text { Unidade } \\
\text { Amostral }\end{array}$ & 1 & 2 & 3 & 4 \\
\hline $\mathrm{R} 2$ & 0.37 & 0.17 & 0.45 & 0.28 \\
\hline $\mathrm{r}$ & -0.61 & -0.41 & -0.67 & -0.52 \\
\hline $\mathrm{p}$-valor $(\mathrm{r})$ & $<2.2 \mathrm{e}-16$ & $<2.2 \mathrm{e}-16$ & $<2.2 \mathrm{e}-16$ & $<2.2 \mathrm{e}-16$ \\
\hline
\end{tabular}

Fonte: os autores.

Há uma correlação negativa moderada entre LST e NDVI para todas as quatro unidades amostrais ( $\mathrm{p}$-valor $\left.<2.2^{\wedge}\{-10\}, \alpha=0.05\right)$. Na unidade amostral 3 , houve melhor relação LST x NDVI, onde $45 \%$ da variável dependente (LST) consegue ser explicada pela variação no NDVI. As demais unidades amostrais (1, 2 e 4) obtiveram valores relativamente baixos de R2 e correlação moderada à fraca conforme classificado por Dancey e Reidy (2006). Os baixos valores de R2 e a moderada correlação observadas nas diferentes unidades amostrais podem estar relacionados ao grande número de outliers presentes nas análises, pois estas foram realizadas através das informações de pixel a pixel, com um grande número de informações presentes para cada unidade avaliada. Outra observação a ser destacada é a diferença de resolução espacial entre as imagens utilizadas no cálculo de NDVI e no cálculo de LST, de 10 metros e 30 metros, respectivamente. Os testes de Shapiro-Wilk (Tab. 3), resultaram em $p>0.005$, implicando que a distribuição dos dados não é significativamente diferente da distribuição normal. Em outras palavras, assume-se a normalidade dos dados. Na Figura 5 pode ser verificado visualmente a normalidade dos dados usando gráficos Q-Q (quantil-quantile plots). O gráfico Q-Q desenha a correlação entre uma dada amostra e a distribuição normal.

Tabela 3: Valores do teste de Shapiro-Wilk para as unidades amostrais.

\begin{tabular}{lcccc}
\hline Unidade Amostral & 1 & 2 & 3 & 4 \\
\hline NDVI & 0.97 & 0.93 & 0.91 & 0.97 \\
\hline LST & 0.89 & 0.88 & 0.85 & 0.96 \\
\hline
\end{tabular}

Fonte: os autores. 
Figura 5: Testes de normalidade de Shapiro-Wilk para cada uma das unidades amostrais.
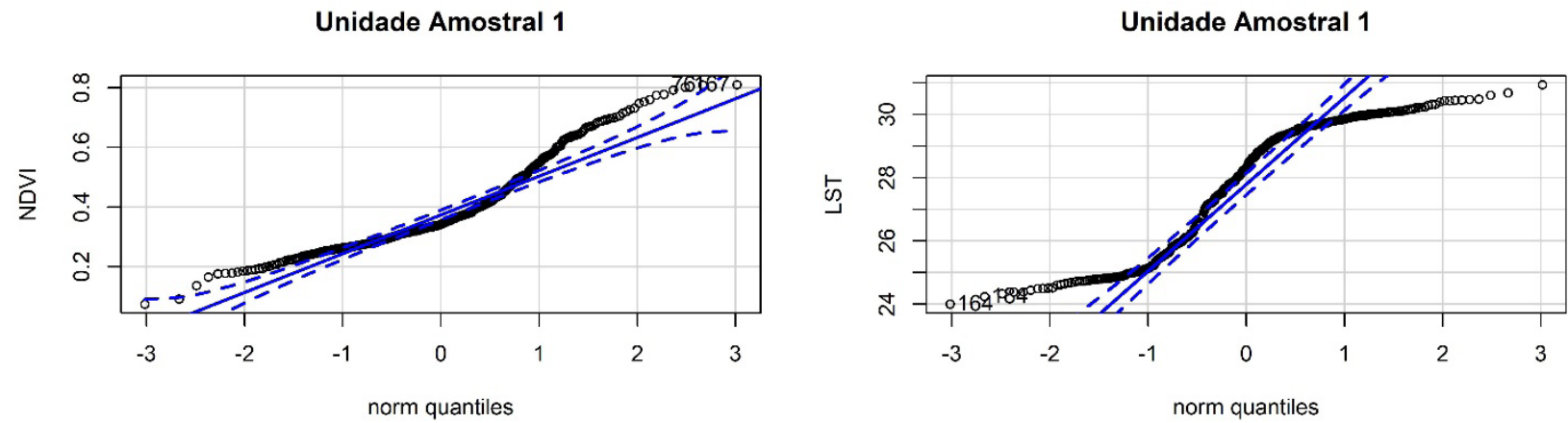

Unidade Amostral 2

Unidade Amostral 2
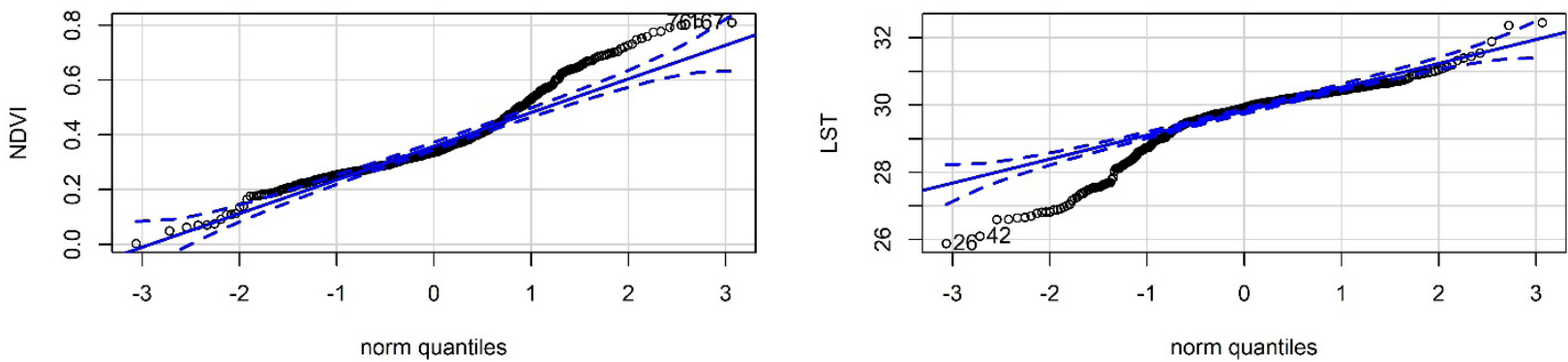

Unidade Amostral 3
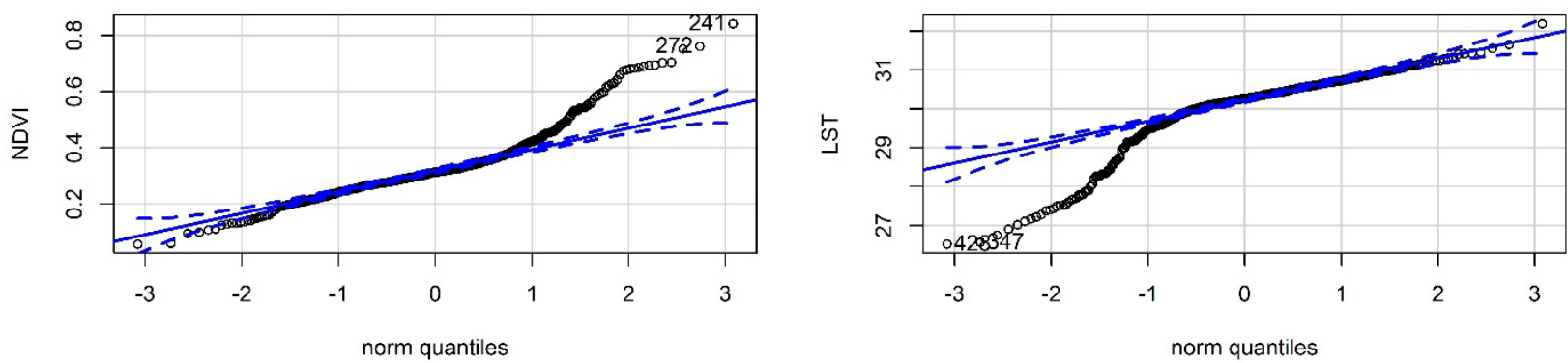

Unidade Amostral 4

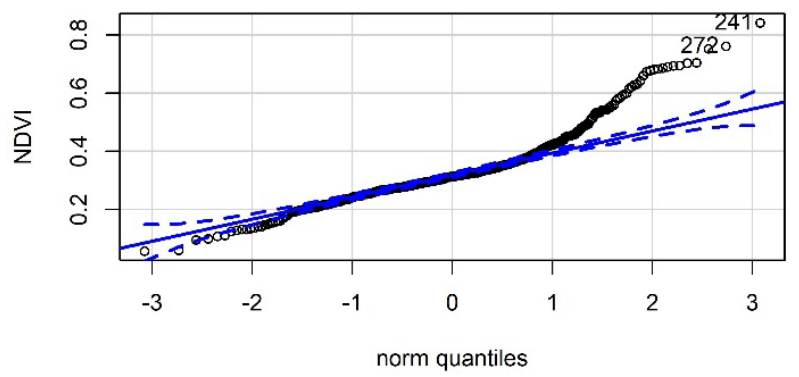

Unidade Amostral 4

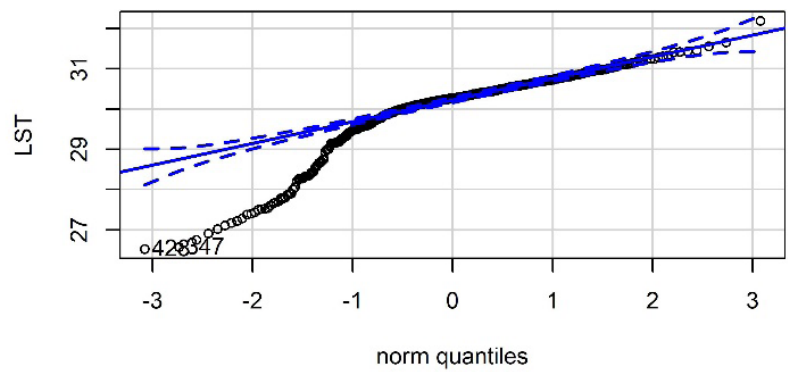

Fonte: os autores. 
Existe forte relação nas variações da temperatura conforme a ausência ou presença de vegetação na superfície terrestre (GORGANI; PANAHI; REZAIE, 2013; YUE et al., 2007; KARNIELI et al., 2010; DENG et al., 2018; MWANGI; KARANJA; KAMAU, 2018). Segundo Yue et al. (2007) o ecossistema urbano conta com um cenário heterogêneo, com alguns parques com altos valores de NDVI, e áreas urbanas construídas ou centrais com baixos valores de NDVI e vegetação relativamente baixa. A distribuição espacial do NDVI na heterogeneidade urbana com diferentes usos e cobertura da terra apresentam valores opostos à LST, com valores de LST significativamente maiores em áreas centrais, e menores em regiões periféricas, locais onde há maior predominância de vegetação.

De maneira geral, os resultados obtidos foram satisfatórios e são passíveis de aplicação na gestão ambiental urbana. A possibilidade de identificar áreas com maiores temperaturas e baixa cobertura vegetal permite agilidade na tomada de decisão e no desenvolvimento de projetos que atenda as peculiaridades de cada setor da cidade, possibilitando a criação de áreas verdes em pontos estratégicos para reduzir a formação de ilhas de calor e amenizar as altas temperaturas nos locais densamente urbanizados, como centros de cidades de grande e médio porte.

A cidade de Rondonópolis é um exemplo de cidade de médio porte que apresenta problemas de má gestão ambiental. O planejamento urbano deve levar em conta meios de garantir a arborização, a criação de parques, a criação de áreas verdes e unidades de conservação para aumentar a qualidade de vida urbana. Por se tratar de uma cidade polo do agronegócio mato-grossense, existe a tendência ao crescimento e expansão da malha urbana, assim como também o aumento da área construída. Esse crescimento deve levar em consideração a importância da preservação de áreas florestais naturais estratégicas, que ajudam na diminuição da temperatura urbana e evitam a formação de ilhas de calor.

\section{CONCLUSÃO}

Neste trabalho, verificou-se moderada correlação negativa entre LST e NDVI, principalmente na área central da cidade, e os picos de LST se dão geralmente em áreas sem cobertura vegetal, como as áreas construídas na região mais central da mancha urbana, enquanto os picos de NDVI aparecem em áreas com vegetação urbana, como parques, praças, chácaras e APPs (neste caso pertencem ao córrego Arareau, na região central urbana, e ao rio Vermelho). Os resultados foram consistentes, portanto, para subsidiar projetos de inserção de vegetação na área urbana, como por exemplo, áreas verdes, parques e praças para a recreação.

A metodologia empregada está ao alcance dos gestores municipais e a compreensão dos efeitos da mudança da cobertura da terra sobre uma área específica, principalmente por área construída, permite a determinação de medidas de mitigação, como a inserção de vegetação em uma grande área com diferentes condições, como um meio de minimizar os efeitos de calor urbano e formação de ilhas de calor. 


\section{AGRADECIMENTOS}

O presente trabalho foi realizado com apoio da Coordenação de Aperfeiçoamento de Pessoal de Nível Superior - Brasil (CAPES) - Código de Financiamento 001.

\section{REFERÊNCIAS}

BEZERRA, P.E.S.; DE MORAES, E.T.I.; SOARES, I.R.C. Análise da Temperatura de Superfície e do Índice de Vegetação no Município de Belém na Identificação das Ilhas de Calor. Rev. Bras. de Cartografia, v.70, n.3, p. 803-818, 2018.

BEZERRA, U.A et al. Comparativo do Índice de Vegetação de Diferença Normalizada (NDVI) entre os Sensores OLI - Satélite Landsat-8 e MSI - Satélite Sentinel-2 em Região Semiárida. Anuário do Instituto de Geociências, v.41, n.3, p. 167-177, 2018.

COLA, C.C.; LOPES, B.B.; BAZAN, W.S. Uso de imagens do Landsat 8 para determinação da correlação entre a temperatura de superfície e o índice de vegetação em municípios da grande Vitória. In: CONGRESSO BRASILEIRO DE CARTOGRAFIA, GEODÉSIA, FOTOGRAMETRIA, E SENSORIAMENTO REMOTO, 27, 2017, Rio de Janeiro, RJ. Anais ... p. 1-5.

DANCEY, C.; REIDY, J. Estatística Sem Matemática para Psicologia: usando SPSS para Windows. Porto Alegre: Artmed, 2006.

DENG, Y. et al. Relationship among land surface temperature and LUCC, NDVI in typical karst area. Scientific Reports, v.8, n. 641, p. 1-12, 2018. DOI: 10.1038/s41598-017-19088-x

GORGANI, S.A.; PANAHI, M.; REZAIE, F. The relationship between NDVI and LST in the urban area of Mashhad, Iran. In: INTERNATIONAL CONFERENCE ON CIVIL ENGINEERING ARCHITECTURE \& URBAN SUSTAINABLE DEVELOPMENT, 2013. Proceedings ...

GODOY, L.B.; BAPTISTA, G.M.M.; ALMEIDA, T. Relação entre vegetação e temperatura de superfície nos parques urbanos do Distrito Federal, por meio de dados ASTER. In: SIMPÓSIO BRASILEIRO DE SENSORIAMENTO REMOTO, 14, 2009, Natal. Anais ...p. 699-705.

KARNIELI, A. et al. Use of NDVI and Land Surface Temperature for Drought Assessment: Merits and Limitations. American Meteorological Society, v. 23, p. 618-632, 2010. Doi: 10.1175/2009JCLI2900.1

LO, C.P.; QUATTROCHI, D.A.; LUVALL, J.C. Application of high-resolution termal infrared remote sensing and GIS to assess the urban heat island effect. International Journal of Remote Sensing, v. 18, n. 2, p. 287-304, 1997. Doi: 10.1080/014311697219079

MWANGI, P.W.; KARANJA, F N.; KAMAU, P.K. Analysis of the Relationship between Land Surface Temperature and Vegetation and Built-Up Indices in Upper-Hill, Nairobi. Journal of Geoscience and Environment Protection, n. 6, 1-16, 2018. Doi: 10.4236/gep.2018.61001

PEEL, M.C.; FINLAYSON, B.L.; MCMAHON, T.A. Updated world map of the Koeppen-Geiger climate classification. Hydrol. Earth Syst. Sci., v.11, p. 1633-1644, 2007.

PINHEIRO, L.C.S.J.; CASTRO, A.S.; MARTINS, E.S. Levantamento das classes de solo existentes nas ecorregiões inseridas no limite do cerrado contínuo. In: SIMPÓSIO NACIONAL DO CERRADO/ SIMPÓSIO INTERNACIONAL SAVANAS TROPICAIS 9,2, Brasília, 2008. Anais ... Brasília: Embrapa, 2008. p. 106-127.

R CORE TEAM. R: A language and environment for statistical computing. R Foundation for Statistical Computing, Vienna, Austria, 2018. Disponível em: https://www.R-project.org/ Acessado em: 10 dez. 2018.

ROUSE, J.W. et al. Monitoring vegetation systems in the Great Plains with ERTS. In: ERTS SYMPOSIUM, 3, 1973. Anais ... NASA SP - 351 I, p. 309-317. 
SMALL, C. The Landsat ETM + spectral mixing space. Remote Sensing of Environment, v.93, p. 1-17, 2004.

SOUZA, A.P. et al. Classificação climática e balanço hídrico climatológico no estado de Mato Grosso. Nativa, n. 1, 34-43, 2013.

USGS. United States Geological Survey. Imagens Sentinel-2. Canais 1, 2, 3, 4, 5, 6, 7, 8, 8A, 9, 10, 11 e 12. Órbita 135, ponto 110. Data de Passagem 14 de maio de 2018. 2018a.

USGS. United States Geological Survey. Imagens Landsat 8. Canais 1, 2, 3, 4, 5, 6, 7 e 8. Órbita 225, ponto 071. Datas de Passagem 20 de maio de 2018. 2018b.

VOOGT, J.A.; OKE, T.R. Thermal Remote Sensing of Urban Climates. Remote Sensing of Environment, $\mathrm{n}$. 86, 370-384, 2003. DOI: 10.1016/S0034-4257(03)00079-8

WILSON, J.S. et al. Evaluating environmental influence of zoning in urban ecosystems with remote sensing. Remote Sensing of Environment, v. 86, p. 303-321, 2003. Doi: 10.1080/01431160500306906

YUE, W. et al. The relationship between land surface temperature and NDVI with remote sensing: application to Shanghai Landsat \& ETM+ data. International Journal of Remote Sensing, v. 28, n. 15, 3205-3226, 2007. Doi: 10.1080/01431160500306906

Data de submissão: 02/ jan./ 2019

Data de aceite: 22/ jan./ 2019 